Pacific Journal of Mathematics

ON TAME CANTOR SETS IN SPHERES HAVING THE SAME ESLIE C. Glaser 


\title{
ON TAME CANTOR SETS IN SPHERES HAVING THE SAME PROJECTION IN EACH DIRECTION
}

\author{
Leslie C. Glaser
}

The main result of this paper is that for each $n \geqq 2$ there exists in $E^{n}$ a tame $(n-1)$-sphere $S$ containing a twice tame Cantor subset $C$ such that all projections of the two sets are the same. That is, if $H_{\alpha}$ is any $(n-1)$-dimensional linear hyperspace in $E^{n}$ and $\pi_{\alpha}: E^{n} \rightarrow H_{\alpha}$ denotes the natural projection of $E^{n}$ onto $H_{\alpha}$, then for every $\alpha$ we have $\pi_{\alpha}(S)=\pi_{\alpha}(C)$. A number of interesting corollaries follow immediately from this result. One corollary is that there exists for each $n \geqq 2$ a countable collection of tame Cantor sets in $E^{n}$ such that each straight line in $E^{n}$ intersects a countable number of these Cantor sets.

1. Introduction and definitions. The main result (Corollaries 1 and 6) of this paper is that for each $n \geqq 2$ there exists in $E^{n}$ a tame $(n-1)$-sphere $S$ containing a twice tame Cantor subset $C$ such that all projections of the two sets are the same (this is made precise in Theorem 1). This generalizes the results of [5], where this result was obtained for $n=3$ and 2 . Our result is obtained by first showing in Theorem 1 that the above result holds for $n \geqq 2$ if we weaken the conclusion by only requiring that $S-C$ is locally flat, rather than $S$ itself being tame. By [3], an immediate corollary to Theorem 1 is the main result for $n \neq 3$ (Corollary 1). We obtain the result for $n=3$ in Corollary 6 by applying [6] and showing that our construction, in fact, produces a 2-sphere having 1-ULC complementary domains. We note that our technique for constructing the desired spheres differs from what was done in [5] for $n=3$. There, an ambient homeomorphism was also constructed along with the desired 2-sphere. The method for constructing the desired Cantor set $C$ is simply a generalization to higher dimensions of the work done in [5], which in turn is actually based on a clever idea of Borsuk in [1]. Interestingly enough, while the work needed to obtain the main result in [5] for $n=3$ is almost as long and involved as our generalization here, an elementary eight line proof of the result when $n=2$ is given in the last paragraph of this same paper. As in [5], we also obtain a number of easy corollaries to our main result. One interesting corollary is that there exists for each $n \geqq 2$ a countable collection of tame Cantor sets in $E^{n}$ such that each straight line in $E^{n}$ intersects a countable number of these Cantor sets.

The organization of our papers is as follows. In the remainder of this section we give some standard definitions. In $\$ 2$, we state our 
basic result, Theorem 1 , and the main result for $n \neq 3$ as Corollary 1. We then note four additional corollaries and their short proofs, assuming the main result for $n \geqq 2$. In $\S 3$, we state two key theorems (Theorems 2 and 3) needed to prove Theorem 1, and then give a proof of Theorem 1 using these two results. In $\S 4$, we prove Theorem 2 . In $\$ 5$, we state and prove a result using a simple canonical geometrical construction which is then used in $\$ 6$, along with Theorem 2, to prove Theorem 3. Finally, in Corollary 6, we show that if we make use of the particularly nice canonical construction suggested by the proof of Theorem 3 to obtain an example satisfying Theorem 1 when $n=3$, then we in fact obtain a tame 2-sphere having the desired properties. We now give some definitions.

By a POLYHEDRON in $E^{n}$, we will mean a compact subset of $E^{n}$ having the same point set as some finite rectilinear subcomplex $K$ of $E^{n}$. Our REGULAR NEIGHBORHOODS will be standard as in [2]. We will call a $k$-sphere $\Sigma$ (or a $k$-cell $D$ ) in $E^{n}$ TAME if there exists an ambient homeomorphism carrying $\Sigma$ (or $D$ ) onto a polyhedron in $E^{n}$. We say a Cantor set $C$ in $E^{n}$ (or $S^{n}$ ) is TAME if there exists a space homeomorphism carrying $C$ into a subset of a line (or a circle). If $C$ is a Cantor set in $S^{k} \subset E^{n}$, then we say that $C$ is TWICE TAME in $S^{k}$ if $C$ is tame in both $S^{k}$ and in $E^{n}$. If $\Sigma$ is a tame $k$-sphere in $E^{n}$ and $H$ is a $(n-k-1)$-hyperplane in $E^{n}$, we say $H$ LINKS $\Sigma$ if $H \cap \Sigma=\phi$ and $\Sigma$ is not homologous to zero in $E^{n}-H$. An open subset $U$ of an $(n-1)$-sphere $S \subset E^{n}$ is LOCALLY FLAT if for any point $x \in U$ there exists an open subset $W$ in $E^{n}$ such that $(W, W \cap U)$ is homeomorphic, as pairs, to $\left(E^{n}, E^{n-1}\right)$. If $H_{\alpha}$ is any $(n-1)$ dimensional linear hyperspace in $E^{n}$, let $\pi_{\alpha}: E^{n} \rightarrow H_{\alpha}$ denote the natural projection of $E^{n}$ onto $H_{\alpha}$. That is, if $L$ is a straight line orthogonal to $H_{\alpha}$, then $\pi_{\alpha}(L)=L \cap H_{\alpha}$. Finally, we give an important definition which will be used in one of our key theorems, Theorem 2 . Given $X \subset E^{n}$ and $\epsilon>0$, let $X^{\epsilon}=\{x \in X \mid d(x, \operatorname{Fr} X) \geqq \epsilon\}$. We note that $X^{\epsilon}$ can also be expressed as $\cup\{x \in X \mid N(x, \epsilon) \subset X\}$. Hence, $X^{\epsilon}$ is a closed subset of int $X$.

\section{Main results.}

THEOREM 1. For each $n \geqq 2$, there exists in $E^{n}$ an $(n-1)$-sphere $S$ containing a twice tame Cantor subset $C$ such that $S-C$ is locally flat and all projections of the two sets are the same. That is, for every $\pi_{\alpha}: E^{n} \rightarrow H_{\alpha}, \pi_{\alpha}(S)=\pi_{\alpha}(C)$.

Applying [3], we immediately obtain the following result.

COROLlaRY 1. For each $n \geqq 4$ or $n=2$, there exists in $E^{n}$ a tame $(n-1)$-sphere $S$ containing a twice tame Cantor subset $C$ such that all projections of the sets are the same. 
For the remaining corollaries, we will assume our main result for all $n \geqq 2$. That is, we will assume Theorem 1 and Corollary 6 , and hence have the analogous result to Corollary 1 for all $n \geqq 2$.

COROLlARY 2. For each $n \geqq 3$, there exists in $E^{n}$ a tame $k$-sphere $\Sigma^{k}(1 \leqq k \leqq n-2)$ which can not be linked by any $(n-k-1)$-hyperplane H.

COROLlary 3. Let $n \geqq 2$, and let $P$ be any subcomplex of $E^{n-1}$ of dimension $\geqq 1$. Then there exists an embedding $h: P \rightarrow E^{n}$ and $a$ Cantor subset $C$ of $P$ such that all projections of $h(P)$ and $h(C)$ are the same. Furthermore, we can pick $h$ and $C$ so that $C$ is tame in $P$ and in $E^{n-1}, h(C)$ is tame in $E^{n}$, and for each simplex $\sigma$ of $P, h(\sigma)$ is tame in $E^{n}$.

COROLLARY 4. If we restrict the above two corollaries to the case where $n \geqq 3, k=n-2$, and $P$ is an $(n-2)$-sphere, then for any tame codimension two knot $K^{n-2} \subset E^{n}$, we obtain the above two conclusions with $h(P)=\Sigma^{n-2}$ embedded equivalently to $K$.

COROLlary 5. There exists a countable collection of tame Cantor sets in $E^{n}(n \geqq 2)$ such that each straight line interval in $E^{n}$ intersects a countable number of these Cantor sets.

Proof of 2. Let $S$ and $C$ be as in Corollary 1 (recall we are assuming Corollary 6 if $n=3$ ). Since $C$ is twice tame, there exists a homeomorphism $f: E^{n} \rightarrow E^{n}$ such that $f(S)$ is a polyhedron and $f(C)$ lies in a line segment in $f(S)$. Given $k, 1 \leqq k \leqq n-2$, let $\hat{\Sigma}$ be a subpolyhedral $k$-sphere in $f(S)$ containing $f(C)$. Let $\Sigma=$ $f^{-1}(\hat{\Sigma})$. Then $C \subset \Sigma \subset S$ and clearly $\Sigma$ is tame. Suppose $H$ is an $(n-k-1)$-hyperplane missing $\Sigma$. Since $H$ then misses $C$ and every projection of $C$ and $S$ are the same, $H$ misses $S$. Since $S$ is tame, int $S$ is an $n$-cell missing $H$. Therefore, $\Sigma$ bounds a $(k+1)$-cell in int $S$ missing $H$, and $H$ does not link $\Sigma$.

Proof of 3. Let $S$ and $f$ be as above. For notational purposes, denote the above $C$ by $\hat{C}$. Since $P$ has dimension $\geqq 1$, we can piecewise linearly embed $P$ in $f(S)$ so that the image of some segment of $P$ contains $f(\hat{C})$. Denote the embedding by $g: P \rightarrow f(S)$. Define $h: P \rightarrow E^{n}$ by $h=f^{-1} \circ g$. Let $C=h^{-1}(\hat{C})$. Since $\hat{C} \subset h(P) \subset S$ and $\pi_{\alpha}(S)=\pi_{\alpha}(\hat{C})$ for all $\alpha$, we have $\pi_{\alpha}(h(P))=\pi_{\alpha}(h(C))$. Clearly, the remaining conclusions also hold.

Proof of 4. Take $P$ in the above proof to be a polyhedral $(n-2)$-disk $D$ and let $g: D \rightarrow f(S)$ be the above map, where $g(D)$ lies 
in some $(n-1)$-simplex $\sigma$ of $f(S)$. Given the knot $K$, in a neighborhood of $\sigma$ in int $f(S)$ attach a polyhedral $(n-2)$-disk $E$ to Bd $g(D)$ so that $E \cap f(S)=\mathrm{Bd} g(D)$ and $E \cup g(D)$ is a polyhedral $(n-2)$-sphere belonging to the same knot class as $K$. Let $F$ be a homeomorphism of $E^{n}$ onto itself carrying $K$ onto $E \cup g(D)$. Define $h: K \rightarrow E^{n}$ by $h=f^{-1} \circ F \mid K$. The result now easily follows. We note, any line intersecting $h(K)$ also intersects $S$, since $h(K) \subset \overline{\operatorname{int} S}$. Therefore, $\pi_{\alpha}(h(K))=\pi_{\alpha}(S)$ for all $\alpha$.

Proof of 5. Let $S$ and $C$ be as in Corollary 1, and let $x_{0} \in$ int $S$. Let $Z$ denote the subset of $E^{n}$ consisting of all points having rational coordinates. Given $z \in Z$, let $T_{z}: E^{n} \rightarrow E^{n}$ be the translation carrying $x_{0}$ to $z$. Then $\left\{T_{z}(C) \mid z \in Z\right\}$ is the desired countable collection of Cantor sets. Let $\epsilon>0$ be so small that $N\left(x_{0}, \epsilon\right) \subset$ int $S$. Given a straight line $L$, pick $z \in Z$ so that $\operatorname{dist}(z, L)<\epsilon$. Then $T_{z}(N(x, \epsilon)) \cap L \neq \phi$, and hence $L \cap T_{z}(S) \neq \phi$. But this implies that $L \cap T_{z}(C) \neq \phi$. Clearly, for any $L$, there are a countable number of such $z$ 's.

3. Proof of Theorem 1. The idea for the following theorem resulted from trying to understand the basic lemmas of [5] so as to isolate and state precisely the key ingredient which would allow us to generalize the results of [5] to higher dimensions.

TheORem 2. Suppose $\delta$ is a positive number, $D$ is a polyhedral $n$-cell in $E^{n}(n \geqq 2)$, and $\tilde{B}_{1}, \tilde{B}_{2}, \cdots, \tilde{B}_{m}$ is a finite collection of polyhedral $n$-cells having disjoint interiors such that $\bigcup_{j=1}^{m} \tilde{B}_{j}=D$, and for each $j=1, \cdots, m$, diam $\tilde{B}_{j}<\delta$. If $\epsilon$ and $\eta$ are positive numbers, $T$ is $a$ (rectilinear) triangulation of $D$ containing each $\tilde{B}_{j}$ as a subcomplex, $Z_{1}$ is a compact subset of $D$ missing the $(n-2)$-skeleton of $T$, and $Z_{2}$ is a compact subset of $D$ missing $\mathrm{Bd} D$, then there exist a finite collection, $B_{i}, B_{2}, \cdots, B_{m}$ of polyhedral $n$-cells and $a$ PL $\eta$-homeomorphism $h$ of $D$ onto itself such that

(i) $h$ carries each $\tilde{B}_{j}$ onto $B_{j}$ and is the identity on $Z_{1} \cup Z_{2} \cup \mathrm{Bd} D$ and, $n$ all $\tilde{B}_{j}$ missing $\operatorname{Bd} D$,

(ii) $\bigcup_{j=1}^{m} B_{j}=D$,

(iii) $\operatorname{diam} B_{j}<\delta$, for $j=1,2, \cdots, m$, and

(iv) there exists a $\gamma>0$ such that $\cup_{j=1}^{m}\left(B_{j}^{\gamma}\right)$ intersects any straight line intersecting $D^{\epsilon}$. In fact, any straight line intersecting $D^{\epsilon}$ intersects some $B_{j}^{\gamma}$ where $B_{j} \cap \mathrm{Bd} D \neq \phi$.

To prove Theorem 1 , we will start with the rectilinear $(n-1)$ sphere $\mathrm{Bd}\left([-1,1]^{n}\right)$ in $E^{n}$. We then will define a sequence of PL homeomorphisms each modifying in elementary canonical fashion the 
previously given polyhedral $(n-1)$-sphere having certain "nice" properties into a new polyhedral $(n-1)$-sphere having similar "nice" properties. An application of Theorem 2 and then a sequence of applications of Theorem 3 (whose proof also will make use of Theorem 2 ) will then give us our desired Cantor set and $(n-1)$-sphere. The next result, Theorem 3, describes what "nice" is and how we do these modifications. Before stating this result, we first need two simple definitions.

Suppose $F$ is a polyhedral $n$-cell in $E^{n}$ and $G$ is a subpolyhedron of $F$. We will call the pair $(F, G)$ a codimension-one cell-cell pair if $(F, G)$ is $\mathrm{PL}$ homeomorphic, as pairs, to $\left([-1,1]^{n-1} \times[-1,1],[-1,1]^{n-1} \times\right.$ $0)$. We will call the pair $(F, G)$ a codimension-one produce cell-sphere pair if $(F, G)$ is PL homeomorphism, as pairs, to

$$
\left([-2,2]^{n-1} \times[-1,1], \operatorname{Bd}\left([-1,1]^{n-1}\right) \times[-1,1]\right) .
$$

Since we will only be considering codimension-one pairs here, for notational purposes we will drop the "codimension-one" and simply denote each of the above pairs as a c.c. pair or a p.c.s. pair. The dimension $n$ will always be clear from the context.

THeorem 3. Let $S$ be a polyhedral $(n-1)$-sphere in $E^{n}(n \geqq 2), X$ a compact subset of $E^{n}$ containing $S$ in its interior, $\epsilon^{\prime}$ is a positive number, $B_{1}, B_{2}, \cdots, B_{m}$ a disjoint collection of polyhedral n-cells, and $G_{1}, G_{2}, \cdots, G_{r}$ a disjoint collection of polyhedral $(n-1)$-cells in $S$ such that

(1) $S-\bigcup_{i=1}^{r}$ int $G_{i}$ misses $\bigcup_{j=1}^{m} B_{j}$,

(2) for each $j, B_{j}$ intersects one and only one $G_{i}$, each $G_{i}$ is interesected by some $B_{j}$, and if $B_{j} \cap G_{i} \neq \phi$, then $\left(B_{i}, B_{j} \cap G_{i}\right)$ is a c.c. or p.c.s. pair, and

(3) any straight line intersecting $X$ also intersects some $B_{j}^{\epsilon^{\prime}}$.

Then, given $\delta_{1}$ and $\delta_{2}>0$ and $0<\eta<\operatorname{dist}\left(S, E^{n}-X\right)$, there exist

(4) positive number $\gamma$ and $\epsilon$, with $0<\epsilon \leqq \epsilon^{\prime}$,

(5) for each $j=1,2, \cdots, m$, a finite collection of disjoint polyhedral $n$-cells $B_{j}, B_{i 2}, \cdots, B_{j m_{j}}$ in int $B_{i}$,

(6) for each $i=1,2, \cdots, r$, a finite collection of disjoint polyhedral $(n-1)$-cells $G_{i 1}, G_{i 2}, \cdots, G_{i r_{i}}$ in int $G_{i}$, and

(7) a PL homeomorphism $f: E^{n} \rightarrow E^{n}$ such that

(8) $\operatorname{diam} B_{j k}<\delta_{1}$ and $\operatorname{diam} G_{i l}<\delta_{2}$,

(9) $f=i d$ on $\left(E^{n}-\bigcup_{j=1}^{m} B_{j}\right) \supset S-\cup_{i=1}^{r}$ int $G_{i}$ and moves points less than $\eta$ on $\cup_{i=1}^{r} G_{i}-\cup_{i=1}^{r} \bigcup_{i=1}^{r_{i}}$ int $G_{i l}$,

(10) $f\left(S-\bigcup_{i=1}^{r} \bigcup_{i=1}^{r_{i}} G_{i l}\right)$ misses $\bigcup_{j=1}^{m} \bigcup_{k=1}^{m_{1}} B_{j k}$, 
(11) for each j, $k, B_{j k}$ intersects one and only one $f\left(G_{i l}\right)$, each $f\left(G_{i l}\right)$ is intersected by some $B_{j k}$, and

each such nonempty intersection is either a c.c. or p.c.s. pair,

(12) for each $G_{i j} \subset \operatorname{int} G_{i}$, there exists an integer $k$ such that $G_{i j}$ and $f\left(G_{i j}\right)$ each lie in int $B_{k}$,

(13) $f(S) \subset \operatorname{int}\left[X \cup\left(\cup_{j=1}^{m} B_{j}^{\epsilon}\right)\right]$ (recall $\left.0<\epsilon \leqq \epsilon^{\prime}\right)$,

(14) any straight line intersecting $\bigcup_{j=1}^{m} B_{j}^{\epsilon}$ also intersects $\bigcup_{i=1}^{m} \bigcup_{k=1}^{m_{j}} B_{j k}^{\gamma}$, and

(15) any straight line intersecting $X \cup\left(\cup_{j=1}^{m} B_{j}^{\epsilon}\right)$ intersects some $B_{j k}^{\gamma}$. (Hence any straight line interesting $f(S)$ also intersects some $B_{j k}^{\gamma}$.)

We now give a proof of Theorem 1 assuming Theorems 2 and 3. Theorem 2 will be proved in $\$ 4$ and Theorem 3 in $\$ 6$.

Proof of Theorem 1. Let $S_{0}=\mathrm{Bd}\left([-1,1]^{n}\right)$ and let $B_{0}=$ $[-2,2]^{n}$. Let $T_{0}$ be any rectilinear triangulation of $B_{0}$ obtained by first triangulating $\mathrm{Bd} B_{0}$ and then letting simplexes of $T_{0}$ be those in $\mathrm{Bd} B_{0}$, the origin, and those of the form the origin joined with a simplex in Bd $\left.B_{0}\right\}$. We note, each $n$-simplex $\Delta$ of $T_{0}$ intersects $S_{0}$ and $\left(\Delta, \Delta \cap S_{0}\right)$ is a c.c. pair. Let $\Delta_{1}, \cdots, \Delta_{m}$ denote the $n$-simplexes of $T_{0}$. Let $\epsilon_{0}$ and $\eta$ each equal $\frac{1}{4}$. We now apply Theorem 2 using $\delta=3, D=B_{0}, \tilde{B}_{i}=\Delta_{i}$, $\epsilon=\epsilon_{0}=\frac{1}{4}, \eta=\frac{1}{4}, T=T_{0}, Z_{1}=\phi$ and $Z_{2}=\left[\frac{-3}{2}, \frac{3}{2}\right]^{n}$. Let $B_{1}, B_{2}, \cdots, B_{m}$ be the collection of polyhedral $n$-cells promised by Theorem 2 . Since $S_{0} \subset Z_{2}$, it follows that $B_{j} \cap S_{0}=\Delta_{j} \cap S_{0}$ for each $i$. Hence, each $B_{j}$ intersects $S_{0}$ and $\left(B_{j}, B_{j} \cap S_{0}\right)$ is a c.c. pair. Also, since each $\Delta_{j}$ has an $(n-1)$-face in $\mathrm{Bd} D$, each $B_{j}$ intersects $\mathrm{Bd} D$. By Theorem 2, (iv) there exists an $\tilde{\epsilon}_{1}\left(=\gamma\right.$ there) such that $\bigcup_{j=1}^{m} B_{j 1}^{\tilde{\epsilon}}$ intersects any straight line intersecting $B_{0}^{\epsilon_{0}}$. Let $X_{1}=B_{0}^{\epsilon_{0}}$ and let $B_{11}, B_{12}, \cdots, B_{1 m 1}$ be polyhedral $n$-cells in the interior of $B_{1}, B_{2}, \cdots, B_{m}$, respectively, such that $B_{j 1}^{\tilde{c}} \subset$ int $B_{i j}$ and $\left(B_{i j}, B_{i j} \cap S_{0}\right)$ is a c.c. pair. (This just requires a small shrinking of each $B_{i}$ using the collar structure of the c.c. pair.) The $B_{i j}$ 's are now disjoint. Choose $\epsilon_{1}^{\prime}>0$ so that $B_{j}^{\epsilon_{1}} \supset B_{j}^{\tilde{\epsilon}_{1}}$. Also, let $G_{11}, G_{12}, \cdots, G_{1 m}$ be disjoint $(n-1)$-cells in $S_{0}$ so that int $G_{1 j} \supset B_{1 j} \cap$ $S_{0}$. Hence $S_{0}-\bigcup_{j=1}^{m} G_{1 j}$ misses $\bigcup_{j=1}^{m} B_{1 j}$. For notational purposes, we now denote $S_{0}$ by $S_{1}$. We observe that the collection $S_{1}, X_{1}, \epsilon_{1}^{\prime},\left\{B_{1 j}\right\}$ and $\left\{G_{1 j}\right\}$ now satisfy the hypothesis of Theorem 3 .

Inductively, suppose that for some fixed $i_{0} \geqq 1$, we have for each $1 \leqq i \leqq i_{0}$ a collection $S_{i}, X_{i}, \epsilon_{i}^{\prime},\left\{B_{i j}\right\}$ and $\left\{G_{i j}\right\}$ satisfying the hypothesis of Theorem 3, and a PL homeomorphism $f_{i-1}: E^{n} \rightarrow E^{n}$ such that $f_{i-1}\left(S_{i-1}\right)=S_{i}, \quad \operatorname{diam} B_{i j}<\frac{3}{2^{i-1}}, \quad$ and $\quad \operatorname{diam}\left[\left(f_{i-1} \circ \cdots \circ f_{1} \circ f_{0}\right)^{-1}\left(G_{i j}\right)\right]<$ $2 / 2^{i-1}$. Also, for $1<i \leqq i_{0}$, we suppose that

$\cup f_{i-1}^{-1}\left(G_{i j}\right) \subset \cup \operatorname{int} G_{i-1, j}, \cup B_{i j} \subseteq \cup \operatorname{int} B_{i-1, j}, X_{i}=X_{1} \cup\left[\bigcup_{k=1}^{i-1} \bigcup_{i=1}^{m_{k}} B_{k_{i}^{k}}^{\varepsilon_{k}}\right]$ 
for some $0<\epsilon_{k} \leqq \epsilon_{k}^{\prime}, f_{i-1}=i d$ on

$$
\left(E^{n}-\bigcup_{j=1}^{m_{i-1}} B_{i-1, j}\right) \supset\left(S_{i-1}-\bigcap_{j=1}^{r_{j}} \text { int } G_{i-1, j}\right)
$$

and for each $G_{i j}$, there exist integers $k$ and $l$ so that $f_{i-1}^{-1}\left(G_{i j}\right)$ lies in (int $\left.G_{i-1, k}\right) \subset\left(\right.$ int $\left.B_{i-1, l}\right)$ and $G_{i j} \subset$ int $B_{i-1, l}$.

We observe, if we let $f_{0}=$ identity, then we have our inductive step for $i_{0}=1$ (the "also" part is vacuous for $i_{0}=1$ ). Now suppose we have our inductive hypothesis for $i_{0}=p$. We will show that the inductive hypothesis holds for $i_{0}=p+1$. Suppose we now have a collection $S_{p}$, $X_{p}, \epsilon_{p}^{\prime},\left\{B_{p j}\right\}_{j=1}^{m_{p}}$ and $\left\{G_{p i}\right\}_{i=1}^{r_{p}}$ satisfying the hypothesis of Theorem 3. Let $\delta_{1}=3 / 2^{p}$ and choose $\delta_{2}$ so small that for any subset $A \subset S_{p}$ of diameter $<\delta_{2}$, we have $\operatorname{diam}\left[\left(f_{p-1} \circ \cdots \circ f_{1} \circ f_{0}\right)^{-1}(A)\right]<2 / 2^{p}$. We now apply Theorem 3 using these $\delta$ 's. The $\gamma$ of Theorem 3, (4) becomes our new $\epsilon_{p+1}^{\prime}$ and the $\epsilon$ our new $\epsilon_{p}$. The $n$-cells of (5) become our collection $\left\{B_{p+1, j}\right\}_{j=1}^{m_{p+1}}$ and the concluded $f$ of (7) is our desired $f_{p}$. We let $S_{p+1}=f_{p}\left(S_{p}\right)$ and the images of the $(n-1)$-cells of (6) under $f_{p}$ become our collection $\left\{G_{p+1, i}\right\}_{i=1}^{r_{p+1}}$. Clearly the diameters of our new $B$ 's and $G$ 's are of the appropriate size. Clearly (5) and (6) of Theorem 3 give us the first two requirements of the "also" part of our induction hypothesis for $i=p+1$. We let $X_{p+1}=X_{p} \cup\left(\cup_{j=1}^{m_{p}} B_{p p}^{\epsilon_{p}}\right)$. This then satisfies the next statement of the "also" part and by (13) of Theorem $3, S_{p+1} \subset$ int $X_{p+1}$. By (15) of that theorem, any straight line intersecting $X_{p+1}$ also intersects some $B_{p+1, j}^{\hat{c}_{p+1}}$. Adding conclusions (10) and (11), we see that our new collection $S_{p+1}, X_{p+1}, \epsilon_{p+1}^{\prime},\left\{B_{p+1, j}\right\}$ and $\left\{G_{p+1, j}\right\}$ also satisfies the hypothesis of Theorem 3. Conclusions (9) and (12) of Theorem 3 give us the final two statements of the "also" part of our inductive hypothesis for $i=p+1$.

Hence, inductively, we obtain for each $i \geqq 1$ a PL homeomorphism $f_{i-1}: E^{n} \rightarrow E^{n}$ such that $f_{i-1}\left(S_{i-1}\right)=S_{i}$, with each $f_{i-1}$ modifying the previous $S_{i-1}$ as described above in the inductive hypothesis. For each $i \geqq 1$, let $h_{i}=f_{i} \circ \cdots \circ f_{1} \mid S_{1}$; recall $S_{1}=\operatorname{Bd}\left([-1,1]^{n}\right)$. We ignore $f_{0}$, since it is the identity. Since the diameters of our $B_{i j}$ 's are less than $3 / 2^{i-1}$, and $f_{i}=i d$ outside the $B_{i j}$ 's, we have that $d\left(h_{i}(x), h_{i-1}(x)\right)<3 / 2^{i-1}$ for all $x \in S_{1}$. Hence $h=\lim _{i \rightarrow \infty} h_{i}$ is a continuous function carrying $S_{1}$ into $E^{n}$.

We recall that $f_{i}$ only modifies $S_{i}$ on the union of the $G_{i j}$ 's. Hence, if we let $H_{i j}=h_{i-1}^{-1}\left(G_{i j}\right)$ for all $i$ and $j$, then for $k \geqq i$ we have that $h_{k}=h_{i-1}$ on $S_{1}-\bigcup_{j=1}^{r_{i}} H_{i j}$. We recall that in defining $f_{i}$ we picked the $G_{i+1, j}$ 's so that $\operatorname{diam} H_{i+1, j}<2 / 2^{i}$ for all $i$ and $j$. Since for each $i$, the collection $\left\{H_{i j}\right\}$ is a disjoint collection of polyhedral $(n-1)$-cells in $S_{1}$ and $\cup H_{i+1, j} \subset \cup$ int $H_{i j}$ (by the first part of the "also" inductive hypothesis) it follows that $C^{*}=\bigcap_{i=1}^{\infty} \cup_{j} H_{i j}$ is a tame Cantor set in $S_{1}$ 
[4]. By similar reasoning, $C=\bigcap_{i=1}^{\infty} \bigcup_{j} B_{i j}$ is also a tame Cantor set in $E^{n}$.

We now claim that $h$ is $1-1$ on $S_{1}$ and that $h\left(C^{*}\right)=C$. We already have observed that $h$ is $1-1$ on $S_{1}-C^{*}$, since each $h_{i}$ is a homeomorphism and for any $x \in S_{1}-C^{*}$, there exists an $i$ such that $h=h_{i}$ in a neighborhood of $x$. Also, since $S_{i}-\cup G_{i j}$ misses $\cup B_{i j}$, for every $i$, no $x \in S_{1}-C^{*}$ is carried to a point of $C$. It remains to show that $h \mid C^{*}$ is a homeomorphism of $C^{*}$ onto $C$. Given any $G_{i j}$, there exist integers $k$ and $l$ so that $f_{i-1}^{-1}\left(G_{i j}\right) \subset$ (int $\left.G_{i-1, k}\right) \cap$ (int $\left.B_{i-1, l}\right)$ and $G_{i j} \subset$ int $B_{i-1, l}$. Therefore $h_{i-1}\left(H_{i j}\right) \subset$ int $B_{i-1, l}$. Since $h_{k}=i d$ outside $\cup B_{i-1, j}$ for $k \geqq i-1$, it follows that $h\left(H_{i j}\right) \subset B_{i-1, l}$. Similarly, given $H_{i+1, k} \subset H_{i j}$, there exists a $B_{i, s}$ such that $h\left(H_{i+1, j}\right) \subset B_{i, s}$. Since $h$ is continuous, $B_{i, s} \subset B_{i-1, l \cdot}$ Since each point of $C^{*}$ is the intersection of $H_{i j}$ 's $i=$ $1,2, \cdots$, and each point of $C$ is the intersection of $B_{i j i}$ 's, it follows that for every $x \in C^{*}, h(x) \in C$. We see that $h$ is $1-1$ on $C^{*}$ because each $B_{i j}$ intersects one and only one $G_{i k} . \quad h \mid C^{*}$ is onto because each $G_{i k}$ is intersected by some $B_{i j}$. Another way to observe that $h \mid C^{*}$ is onto is that clearly $h\left(S_{1}\right) \supset C$ and no point of $S_{1}-C^{*}$ is carried to $C$.

We now claim that $h\left(S_{1}\right)=S \supset C$ satisfies Theorem 1. Since $h\left(C^{*}\right)=C, C$ is twice tame in $S$. To prove that all projections of $C$ and $S$ are the same, it suffices to show that any straight line $L$ intersecting $S$ also intersects $C$. Hence, suppose we are given $L$, such that $L \cap S \neq \phi$. If $L$ does not intersect $C$ then for some large $i, L$ misses $\bigcup_{j=1}^{m_{i}} B_{i j}$. Since all further modifications of $S_{i}$ occur in $\bigcup_{j=1}^{m_{i}} B_{i j}$ and $L \cap S \neq \phi$, it follows that $L \cap S_{i} \neq \phi$. Now $S_{i} \subset X_{i}$ and any line intersecting $X_{i}$ also intersects some $B_{i j}^{\xi_{i}} \subset B_{i j}$. This contradiction shows that $L \cap C \neq \phi$, and this completes the proof of Theorem 1 .

4. Proof of Theorem 2. Given $D$ and $\bigcup_{j=1}^{m} \tilde{B}_{i}=D$ such that diam $\tilde{B}_{j}<\delta$, suppose $T$ is a triangulation of $D$ containing each $\tilde{B}_{j}$ as a subcomplex and $Z_{1}$ is a compact subset of $D$ missing the $(n-2)$ skeleton of $T$. Given $\epsilon$ and $\eta>0$, we may suppose by taking smaller numbers if necessary (certainly, if (iv) holds for a smaller $\epsilon^{\prime}$, it will hold for the given one-since if $0<\epsilon^{\prime} \leqq \epsilon$, then $\left.D^{\epsilon} \subset D^{\epsilon^{\prime}}\right)$ that $D^{\epsilon} \supset Z_{2}$ $D^{\epsilon} \supset Z_{2} \cup\left\{\tilde{B}_{j} \mid \tilde{B}_{j} \cap \operatorname{Bd} D=\phi\right\} \cup\{$ simplexes $\Delta \in T \mid \Delta \cap \operatorname{Bd} D=\phi\}$ and that $\eta<1 / 4 \min \left(\left\{\delta-\operatorname{diam} \tilde{B}_{j} \mid j=1,2, \cdots, m\right\}\right.$, dist $\left.\left(Z_{1}, T^{(n-2)}\right)\right)$, where $T^{(n-2)}$ is the $(n-2)$-skeleton of $T$. Since $T$ contains each $\tilde{B}_{j}$ as a subcomplex and $\bigcup_{j=1}^{m} \tilde{B}_{j}=D$, it follows that the mesh of $T<\delta$.

Let $\Delta_{1}, \cdots, \Delta_{r}$ be the $n$-simplexes of $T$, ordered so that the $n$-simplexes of $T$ having faces in $\operatorname{Bd} D$ appear first in this ordering. That is, suppose each of $\Delta_{1}, \cdots, \Delta_{t}$ intersects $\mathrm{Bd} D$, while each of $\Delta_{t+1}, \cdots, \Delta_{r}$ lie in int $D$. By our assumption on $\epsilon, \Delta_{t+1} \cup \cdots \cup$ $\Delta_{r} \subset D^{\epsilon}$ and given any $\Delta_{i}(1 \leqq i \leqq t)$, then each face of $\Delta_{i}$ missing $\operatorname{Bd} D$ also lies in $D^{\epsilon}$. We will now consider each $\Delta_{1}$ through $\Delta_{t}$ in turn, and 
construct small "ridges" on them so that if $R_{i}$ denotes the ridge constructed on $\Delta_{i}$, then $\Delta_{i} \cup R_{i}$ is a polyhedral $n$-cell, each $R_{i}$ misses $D^{\epsilon} \cup \operatorname{Bd} D \cup Z_{1}$ (hence misses $Z_{2}$, since $Z_{2} \subset D^{\epsilon}$ ), the $R_{i}$ 's are disjoint, and $R_{i} \cap \operatorname{Bd} \Delta_{i}$ separates $\operatorname{Bd} \Delta_{i} \cap D^{\epsilon}$ from $\operatorname{Bd} \Delta_{i} \cap \operatorname{Bd} D$ (in $\operatorname{Bd} \Delta_{i}$ ). Also, each ridge $R_{i}$ will be so small that if $\sigma$ is any simplex of $T$ missing $\Delta_{i}$, then $R_{i} \cap \sigma=\phi$. Actually, each $R_{i}$ will be PL equivalent to $N_{i} \times\left[-\frac{1}{2}, \frac{1}{2}\right] \times\left[0, \frac{1}{2}\right]$, where $N_{i}$ is a polyhedral $(n-2)$-manifold in $\operatorname{Bd} \Delta_{i}$ separating $\mathrm{Bd} \Delta_{i} \cap D^{\epsilon}$ from $\mathrm{Bd} \Delta_{i} \cap \mathrm{Bd} D$ and $R_{i} \cap \Delta_{i} \subset \mathrm{Bd} \Delta_{i}$ will correspond to $N_{i} \times[0,1] \times 0$. Since all the ridges will be of this form and will be disjoint and will all miss $Z_{1} \cup \operatorname{Bd} D \cup D^{\epsilon}$, we can construct for each $i=1,2, \cdots, t$ PL $\eta$-homeomorphism $h_{i}$ taking $D$ onto itself such that $h_{i}$ only moves points in a small neighborhood $W_{i}$ of $R_{i}$ and takes $\Delta_{i}$ onto $\Delta_{i} \cup R_{i}$. The $W_{i}$ 's will be small enough neighborhoods so as to be disjoint and also miss $Z_{1} \cup \operatorname{Bd} D \cup D^{\epsilon}$. The desired $h$ will then be $h_{t} \circ h_{t-1} \circ \cdots \circ h_{1}$ and $B_{i}$ will be defined by $B_{i}=h\left(\tilde{B}_{i}\right)$. Clearly, $h$ is a PL $\eta$-homeomorphism and conclusions (i), (ii) and (iii) follow immediately.

We first consider $\Delta_{1}$. We know $\Delta_{1} \cap \mathrm{Bd} D \neq \phi$. We can assume for each $i, 1 \leqq i \leqq t$, that $\operatorname{Bd} \Delta_{i} \neq \mathrm{Bd} D$. Otherwise $\Delta_{1}=D=\tilde{B}_{1}$ and we can take $h=i d$ and $\gamma=\epsilon$. Let $M_{1}$ be a "small" regular neighborhood of $\Delta_{1} \cap \mathrm{Bd} D$ in $\operatorname{Bd} \Delta_{1}$ so that $M_{1}$ misses $\Delta_{1} \cap D^{\epsilon}$ and so that the various components of $M_{1}$ correspond to regular neighborhoods of the various components of $\Delta_{1} \cap \mathrm{Bd} D$. Also, since $Z_{1}$ misses the $(n-2)$ - skeleton of $T$, if we take a small enough regular neighborhood so that $\mathrm{Bd} M_{1}$ lies in a small neighborhood of $T^{(n-2)}$, we can suppose $B d M_{1}=N_{1}$ misses $Z_{1}$. We now simply repeat the procedure for each $\Delta_{i}(i=2,3, \cdots, t)$ in turn, taking smaller and smaller regular neighborhoods each time to insure that the $N_{i}$ 's ( $=\mathrm{Bd} M_{i}$ 's) are disjoint. (We can think of the $N_{i}$ 's as lying in smaller and smaller neighborhoods of $\mathrm{Bd} D$ as $i$ increases. That is, we can think of $M_{1}$ as being the simplicial neighborhood of $\Delta_{1} \cap \mathrm{Bd} D$ in $\mathrm{Bd} \Delta_{1}$ under some sufficiently high barycentric subdivision of $T$, and the consecutive $M_{i}$ 's will be similar except each lying in higher and higher barycentric subdivisions of $T$.)

Now let each $W_{i}$ be an appropriately "small" regular neighborhood of $N_{i}$ in int $D$ under some sufficiently high further barycentric subdivision of $T$. By small we mean small enough so that the $W_{i}$ 's are disjoint (components of each $W_{i}$ correspond to components of each $N_{i}$ ), $\cup W_{i}$ misses $D^{\epsilon} \cup B d D \cup Z_{1}$, and $\Delta_{i} \cup W_{i}$ lies in an $\eta$-neighborhood of $\Delta_{i}$. It follows easily from standard PL theory ([2]) that each $W_{i} \approx$ $N_{i} \times[-1,1]^{2}$ with $W_{i} \cap \Delta_{i} \approx N_{i} \times[-1,1] \times[-1,0], \quad W_{i} \cap \operatorname{ext} \Delta_{i} \approx$ $N_{i} \times[-1,1] \times[0,1]$, and $W_{i} \cap \operatorname{Bd} \Delta_{i} \approx N_{i} \times[-1,1] \times 0$. Since the PL equivalence can be obtained carrying $x \in N_{i}$ to $(x, 0,0) \in N_{i} \times[-1,1]^{2}$, by taking a smaller $W_{i}$, if necessary, we can suppose that the preimage of $x \times[-1,1]^{2}$ lies in a $\eta$-neighborhood of $x$ in int $D$ for each $x \in$ $N_{i}$. The reçuired $R_{i} \subset$ int $W_{i}$ will then be the regular neighborhood of 
$N_{i}$ in ext $\Delta_{i} \cap$ int $W_{i}$ corresponding to $N_{i} \times\left[-\frac{1}{2}, \frac{1}{2}\right] \times\left[0, \frac{1}{2}\right] \subset N_{i} \times$ $[-1,1]^{2}$. To define the desired $\eta$-homeomorphism $h_{i}: D \rightarrow D$ keeping points of $D$-int $W_{i}$ fixed and carrying $\Delta_{i}$ onto $\Delta_{i} \cup R_{i}$ is now elementary.

As we have already noted, our PL homeomorphism $h$ is $h_{t} \circ h_{t-1} \circ \cdots \circ h_{1}$. We observe that $h$ only moves points in simplexes intersecting $\mathrm{Bd} D$ and given any $\Delta_{i}, 1 \leqq i \leqq t, h\left(\Delta_{i}\right) \supset R_{i} \cup\left(\Delta_{i}-\right.$ [small nbd. of $\left.\left.\cup_{j=1}^{t} i \neq j, R_{j}\right]\right)$, and for $i>t, h\left(\Delta_{i}\right)=\Delta_{i}$.

It now remains to prove cconclusion (iv). We claim that any straight line $L$ intersecting $D^{\epsilon}$ intersects the interior of some $B_{i}=$ $h\left(\tilde{B}_{i}\right)$. Since each $\tilde{B}_{i}$ is the union of certain $\Delta_{j}$ 's, it suffices to show that $L$ intersects the interior of some $h\left(\Delta_{j}\right)$. In fact, we will show that $L$ intersects the interior of some $h\left(\Delta_{j}\right)$ where $h\left(\Delta_{j}\right) \cap \mathrm{Bd} D=$ $\Delta_{j} \cap \operatorname{Bd} D \neq \phi$. Given $L$, there exists a $p \in \operatorname{Bd} D^{\epsilon}$ and a $p^{\prime} \in \operatorname{Bd} D$ such that the open segment $U$ of $L$ between $p$ and $p^{\prime}$ misses $D^{\epsilon} \cup B d D$. If $U$ lies in no proper face of any $n$-simplex $\Delta_{i}$ of $T$, then for some point $p^{\prime \prime}$ of $U$ near $p^{\prime}$, we could have the open segment $V$ of $L$ between $p^{\prime \prime}$ and $p^{\prime}$ lying in the interior of some $\Delta_{i}$. If we pick $p^{\prime \prime}$ close enough to $p^{\prime}$ so as to miss all the ridges and their neighborhoods, the $W_{i}$ 's, then $V \subset h$ (int $\Delta_{i}$ ). If $U$ lies in some proper face of some $\Delta_{i}$, then since $N_{i}$ separates $p$ from $p^{\prime}$, there exists a point $\hat{p}$ of $L$ in $N_{i}$. But then, $\hat{p} \in h$ (int $\Delta_{i}$ ), since $N_{i} \subset h$ (int $\Delta_{i}$ ).

To complete the proof now, we make use of the following argument given in [1], p. 274. Let $\tilde{\Lambda}$ denote the space of all lines $L$ in $E^{n}$. That is, points of $\tilde{\Lambda}$ are straight lines in $E^{n}$. The topology of $\tilde{\Lambda}$ is generated by the following basis. Given $L \in \tilde{\Lambda}$, a finite collection $p_{1}, p_{2}, \cdots, p_{m}$ of points of $L$, and $\epsilon_{1}, \epsilon_{2}, \cdots, \epsilon_{m}>0$, let $N\left(L,\left(p_{1}, p_{2}, \cdots, p_{m}\right),\left(\epsilon_{1}, \cdots, \epsilon_{m}\right)\right\}$ be the set of all lines $\tilde{L}$ in $E^{n}$ such that for each $i$ there exists $\tilde{p}_{i} \in \tilde{L}$ with dist $\left(p_{i}, \tilde{p_{i}}\right)<\epsilon_{i}$. This has a subbasis of the form $\{N(L, p, \epsilon) \mid \in \tilde{\Lambda}$, $p \in L$, and $\epsilon>0\}$. For given any $U=N\left(L,\left(p_{1}, \cdots, p_{m}\right),\left(\epsilon_{1}, \cdots, \epsilon_{m}\right)\right)$ and $\tilde{L} \in U$, then $\bigcap_{i=1}^{m} N\left(\tilde{L}, \tilde{p}_{i}, \tilde{\epsilon}_{i}\right) \subset U$, where $\operatorname{dist}\left(p_{i}, \tilde{p}_{i}\right)=\delta_{i}<\epsilon_{i}$ and $\tilde{\epsilon}_{i} \leqq \epsilon_{i}-\delta_{i}$. We note $\tilde{\Lambda}$ also has a basis of the form $\{N(L(r, s),(\epsilon, \epsilon))\}$ where for each $L \in \tilde{\Lambda}$ we used fixed points $r \neq s \in L$. For given any $U$ as above and $\tilde{L} \in U$, we consider $N\left(\tilde{L}, \tilde{p}_{i}, \tilde{\epsilon}_{i}\right) i=1,2, \cdots, m$ where $\bigcap_{i=1}^{m} N\left(\tilde{L}, \tilde{p}_{i}, \tilde{\epsilon}_{i}\right) \subset U$. Let $r$ and $s$ be any two distinct points of $\tilde{L}$. For each $i$, there exists a $\delta_{i}>0$ so that any $\hat{L} \in N\left(\tilde{L},(r, s),\left(\delta_{i}, \delta_{i}\right)\right)$ intersects the $\tilde{\epsilon}_{i}$-neighborhood of $\tilde{p}_{i}$ in $E^{n}$. Hence, if $\epsilon=\min \left\{\delta_{i} \mid i=1,2, \cdots, m\right\}$, then

$$
N(\tilde{L},(r, s),(\epsilon, \epsilon)) \subset \bigcap_{i=1}^{m} N\left(\tilde{L}, \tilde{p}_{i}, \tilde{\epsilon}_{i}\right) \subset U
$$

Let $\Lambda$ denote the subspace of $\tilde{\Lambda}$ consisting of all lines $L$ in $E^{n}$ intersecting $D^{\epsilon}$. For each point $p \in \operatorname{Bd} D^{\epsilon}$ and $p^{\prime} \in \operatorname{Bd} D$, let $L\left(p, p^{\prime}\right)$ denote the straight line in $E^{n}$ containing $p$ and $p^{\prime}$. Let $F: \operatorname{Bd} D^{c} \times$ 
$\operatorname{Bd} D \rightarrow \Lambda$ be defined by $F\left(p, p^{\prime}\right)=L\left(p, p^{\prime}\right)$. Since $\operatorname{dist}\left(p, p^{\prime}\right) \geqq \epsilon$, $L\left(p, p^{\prime}\right)$ depends continuously on $\left(p, p^{\prime}\right)$. Since $\operatorname{Bd} D^{\epsilon} \times \operatorname{Bd} D$ is compact and $F$ carries this space continuously onto $\Lambda, \Lambda$ is compact. Now for each $L \in \Lambda$, let $r(L)=\sup \left\{s \mid N(x, s) \subset\right.$ some $B_{i}$, with $B_{i} \cap$ $\operatorname{Bd} D \neq \phi$, and $x \in L\}$. By the above paragraph, $r(L)>0$ for all $L \in \Lambda$. Since $r(L)$ depends continuously on $L$, there exists a $\gamma_{0}$ such that $r(L) \geqq \gamma_{0}$ for every $L \in \Lambda$. But then, given any $\gamma, 0<\gamma<\gamma_{0}$, it follows that if $L \in \Lambda$, then $L$ meets $\cup_{i=1}^{m} B_{i}^{\gamma}$. This completes the proof of Theorem 2.

5. A geometrical construction. In order to state our next result, we first need some notation. Let $r D^{n}$ be the polyhedral $n$-cell in $E^{n}$ defined by $r D^{n}=[-r, r]^{n}$ and let $\Sigma^{n-1}$ denote the polyhedral $(n-1)$-sphere in $E^{n}$ bounding $D^{n}=[-1,1]^{n}$. For a given $n \geqq 2$, we are going to want to again consider c.c. and p.c.s. polyhedral pairs. Recall these are polyhedral pairs PL homeomorphic to $\left(D^{n}, D^{n-1}\right)$ or to $\left(2 D^{n-1} \times[-1,1], \Sigma^{n-1} \times[-1,1]\right)$, respectively. Those of the first type divide naturally into two polyhedral $n$-cells and those of the second type divide naturally into three polyhedral $n$-cells as follws. We express $D^{n}$ as $D_{+}^{n} \cup D_{-}^{n}$, where $D_{+}^{n}=D^{n-1} \times[0,1]$ and $D_{-}^{n}=D^{n-1} \times[-1,0]$. We express $2 D^{n-1} \times[-1,1]$ as $F_{0} \cup F_{+} \cup F_{-}$, where $F_{0}=D^{n-1} \times[-1,1]$ and $F_{\alpha}=\left(2 D_{\alpha}^{n-1}-\right.$ int $\left.D^{n-1}\right) \times[-1,1], \alpha=$ +or - . We note $D_{+}^{n} \cap D_{-}^{n}=D^{n-1} \times 0$ (which we have identified with $\left.D^{n-1}\right)$

$$
\begin{gathered}
F_{+} \cup F_{-}=\left(2 D^{n-1}-\text { int } D^{n-1}\right) \times[-1,1], F_{+} \cap F_{-}=\left(2 D^{n-2}-\text { int } D^{n-2}\right) \\
\times[-1,1], F_{0} \cap\left(F_{+} \cup F_{2}\right)=\Sigma^{n-2} \times[-1,1],
\end{gathered}
$$

and $F_{0} \cap F_{\alpha}=\sum_{\alpha}^{n-2} \times[-1,1], \alpha=+$ or - , where $\sum_{\alpha}^{n-2}=\Sigma^{n-2} \cap B_{\alpha}^{n-1}, \alpha=$ tor - .

Given any $m \geqq 0$, let $D_{(m)}^{n}$ and $\left(2 D^{n-1} \times[-1,1]\right)_{(m)}$ denote the "cellular" rectilinear subdivision of $D^{n}$ and $\left(2 D^{n-1} \times[-1,1]\right)$ obtained by considering the union of the cells of the form $\times_{j=1}^{n}\left[i_{i}, k_{j}\right]$ and $\times_{j=1}^{n}\left[s_{j}, t_{j}\right]$, respectively, plus their faces, where $i_{j}(j=1, \cdots, n)$ and $s_{n}$ is of the form $p / 2^{m}$ and $p$ is an integer such that $-2^{m} \leqq p<2^{m}, s_{i}$ $(j=1, \cdots, n-1)$ is of the form $q / 2^{m}$ and $q$ is an integer such that $-2^{m+1} \leqq q<2^{m+1}$, and in each case $k_{j}=i_{j}+1 / 2^{m}$ and $t_{j}=s_{j}+1 / 2^{m}$. We note each of $D_{(m)}^{n}$ and $\left(2 D^{n-1} \times[-1,1]\right)_{(m)}$ contains $D^{n-1}$ and $\Sigma^{n-1} \times$ $[-1,1]$ as a "cellular" subcomplex. Also, for each $m \geqq 1, D_{(m+1)}^{n}$ and $\left(2 D^{n-1} \times[-1,1]\right)_{(m+1)} \quad$ subdivides $D_{(m)}^{n}$ and $\left(2 D^{n-1} \times[-1,1]\right)_{(m)}$, respectively. For each $n \geqq 2$ and $m \geqq 1$, let $\tilde{A}_{+(m)}^{n-1}$ and $\tilde{A}_{-(m)}^{n-1}$ denote the unique disjoint $(n-1)$-cells in $D^{n-1}$ containing the point $(1, \cdots, 1)$ and $(-1,-1, \cdots,-1)$, respectively. For each $n \geqq 2$ and $m \geqq 1$, let $\tilde{C}_{\alpha(m)}^{n-1}$, 
$\tilde{C}_{+(m)}^{n-1}, \tilde{C}_{-(m)}^{n-1}$ denote the disjoint $(n-1)$-cells in $\Sigma^{n-2} \times[-1,1]$ defined by $\left(\tilde{A}_{+(m)}^{n-2} \times 1\right) \times\left[-1,-1+1 / 2^{m}\right], \quad\left(\tilde{A}_{+(m)}^{n-2} \times 1\right) \times\left[1-1 / 2^{m}, 1\right], \quad$ and $\left(\tilde{A}_{-(m)}^{n-2} \times\{-1\}\right) \times\left[-1,-1+1 / 2^{m}\right]$, respectively.

Observation 1. Given any $n \geqq 2$ and $m \geqq 1, D_{(m)}^{n}$ and $\left(2 D^{n-1} \times[-1,1]\right)_{(m)}$ induce "cellular" rectilinear subdivisions of $D_{\alpha}^{n}$ $(\alpha=+$ or -$)$ and $F_{\beta}^{n}(\beta=0,+$ or -$)$. We will denote these by $D_{\alpha(m)}^{n}$ and $F_{\beta(m)}^{n}$, respectively. Moreover, by merely moving back and forth, layer by layer, it is not too difficult to see that the $n$-cells of $D_{\alpha(m)}^{n}$ can be ordered, starting with the one containing $\tilde{A}_{\alpha(m)}^{n-1}$ or $\tilde{C}_{\beta(m)}^{n-1}$, respectively, so that any two consecutive cells of our ordering have an $(n-1)$-face in common. Given such an ordering, let $\tilde{a}_{\alpha(m)}$ (or $\tilde{c}_{\beta(m)}$ ) denote the rectilinear path in $D_{\alpha}^{n}$ (or $F_{\alpha}^{n}$ ) obtained by taking the union of the following segments: the segment joining the barycenter of $\tilde{A}_{\alpha(m)}^{n-1}$ (or $\left.\tilde{C}_{\beta(m)}^{n-1}\right)$ to the barycenter of our first $n$-cell (which contains this given $(n-1)$-cell) and then adding the segments joining the barycenters of our consecutive cells in our ordering. We note, $\tilde{a}_{\alpha(m)}$ (or $\tilde{c}_{\alpha(m)}$ ) minus the barycenter of $\tilde{A}_{\alpha(m)}^{n-1}\left(\right.$ or $\left.\tilde{C}_{\alpha(m)}^{n-1}\right)$ lies in the interior of $D_{\alpha}^{n}$ (or $F_{\alpha}^{n}$ ) and $\tilde{a}_{\alpha(m)}$ (or $\left.\tilde{c}_{\alpha(m)}\right)$ intersects each $n$-cell of $D_{\alpha(m)}^{n}\left(\right.$ or $\left.F_{\alpha(m)}^{n}\right)$ and this intersection is, in fact, a simple spanning path, except in the case of the last $n$-cell involved.

We now can state our desired result.

THEOREM 4. Suppose $S$ is a polyhedral $(n-1)$-sphere in $E^{n}(n \geqq$ 2), $B_{1}, B_{2}, \cdots, B_{m}$ are disjoint polyhedral $n$-cells, and $G_{1}, G_{2}, \cdots, G_{r}$ are disjoint polyhedral $(n-1)$-cells in $S$ such that

(1) $S-\bigcup_{i=1}^{r}$ int $G_{i}$ misses $\bigcup_{j=1}^{m} B_{i}$,

(2) for each $j, B_{j}$ intersects one and only one $G_{i}$ (different $B_{j}$ 's may intersect the same $G_{i}$ )

(3) each $G_{i}$ is intersected by some $B_{j}$, and

(4) if $B_{j} \cap G_{i} \neq \phi$, then there exists a PL homeomorphism $h_{j}$ carrying the polyhedral pair $\left(B_{j}, B_{j} \cap G_{i}\right)$ onto one of the two types described above.

If $h_{j}:\left(B_{j}, B_{j} \cap G_{i}\right) \rightarrow\left(D^{n}, D^{n-1}\right)$ and we let $B_{j \alpha}=h_{j}^{-1}\left(D_{\alpha}^{n}\right)$ and $A_{j \alpha}=$ $h_{j}^{-1}\left(\tilde{A}_{\alpha(1)}\right)(\alpha=+$ or -$)$, or if $h_{k}:\left(B_{k}, B_{k} \cap G_{i}\right) \rightarrow\left(2 D^{n-1} \times[-1,1], \Sigma^{n-2} \times\right.$ $[-1,1])$ and we let $B_{k \beta}=h_{k}^{-1}\left(F_{\beta}\right)$ and $C_{k \beta}=h_{k}^{-1}\left(\tilde{C}_{\beta(1)}\right)(\beta=0$, + or -$)$, then given positive numbers $\epsilon_{1}$ and $\epsilon_{2}$, and a rectilinear triangulation $T$ of $E^{n}$ containing all the above polyhedra as subcomplexes (such a $T$ exists by [2]), there exist

(5) a subdivision $\tilde{T}$ of $T$ such that for each $j \alpha(k \beta)$ there exists a finite collection $B_{j \alpha 1}, B_{j \alpha 2}, \cdots, B_{j \alpha m(j \alpha)}$ of polyhedral $n$-cells having disjoint interiors such that $\cup B_{j \alpha i}=B_{j \alpha}$ and each $B_{j \alpha i}$ is a subcomplex of $\tilde{T}$ of diameter $<\epsilon_{1}$ (the corresponding conclusion holds for each $k \beta$ ), and 
(6) a PL homeomorphism $h: E^{n} \rightarrow E^{n}$ and for each ja $(k \beta)$ there exists a polyhedral $(n-1)$-cell $G_{j \alpha}$ lying in the interior of $A_{j \alpha}$ such that $\operatorname{diam} G_{j \alpha}<\epsilon_{2}, h$ (int $\left.G_{j \alpha}\right) \subset$ int $B_{j \alpha}, h\left(G_{j \alpha}\right)$ misses the $(n-2)$-skeleton of $\tilde{T}$ and intersects each $B_{j \alpha i}(i=1,2, \cdots, m(j \alpha))$ so as to give a polyhedral pair of one of the two types described above (the analogous statements holds for each $k \beta$ where $G_{k \beta} \subset$ int $C_{k \beta}$ ), each of hypothesis (2) and (3) holds for the new "smaller" $B$ 's and $h(G)$ 's, and $h=i d$ on

$$
\left(E^{n}-\bigcup_{j=1}^{m} B_{i}\right) \cup\left[S-\left(\cup \operatorname{int} G_{j \alpha}\right) \cup\left(\cup \operatorname{int} G_{k \beta}\right)\right]
$$

Observation 2. We note, given $G_{i}$, if $G_{i}$ intersects $B_{j 1}, \cdots, B_{j m}$, where for some $k_{0}, 0 \leqq k_{0} \leqq m_{j},\left(B_{j k}, B_{j k} \cap G_{i}\right)$ is of the first type for $1 \leqq k \leqq k_{0}\left(=\phi\right.$ if $\left.k_{0}=0\right)$ and $\left(B_{j k}, B_{j k} \cap G_{i}\right)$ is of the second type for $k_{0}+1 \leqq k \leqq m_{j}\left(=\phi\right.$ if $\left.k_{0}=m_{j}\right)$, then the above result gives us a new collection of disjoint polyhedral $(n-1)$-cells $G_{j k \alpha}\left(1 \leqq k \leqq k_{0}, \alpha=+\right.$ or -$)$ and $G_{j k \beta}\left(k_{0}+1 \leqq k \leqq m_{j}, \beta=0\right.$, +or -$)$ in int $G_{i}$ each lying in the interior of the appropriate $B_{j k}$. Moreover, if we shrink each $B_{j k \alpha i}$ or $B_{j k \beta i}$ (where $j k$ corresponds to some $j$ or $k$ in (5) above) using the appropriate collar structure, we can obtain a disjoint collection of polyhedral $n$-cells $\left\{\tilde{B}_{j k \alpha i}, \tilde{B}_{j k \beta i}\right\}$ so that

$$
S-\left(\cup \operatorname{int} G_{j k \alpha}\right) \cup\left(\cup \operatorname{int} G_{j k \beta}\right)=h\left(S-\left(\cup \operatorname{int} G_{j k \alpha}\right) \cup\left(\cup \operatorname{int} G_{j k \beta}\right)\right.
$$

misses the union of the $\tilde{B}_{j k \alpha i}$ 's and $\tilde{B}_{j k \beta i}$ 's, and each $h\left(G_{j k \alpha}\right) \cap \tilde{B}_{j k \alpha i}$ and $h\left(G_{j k \beta}\right) \cap \tilde{B}_{j k \beta i}$ is a polyhedral pair of one of the two types above. That is, the new collection of $(n-1)$-cells and $n$-cells (the $\tilde{B}_{j k a i}$ 's) are disjoint and satisfy the hypothesis (1)-(4) of Theorem 4.

Proof of Theorem 4. Let $T$ be the triangulation of $E^{n}$ containing all the given polyhedra. Given, the $h_{j}$ and $h_{k}$, choose an integer $M$ large enough so that the preimage of each $n$-cell of $D_{(M)}^{n}$ under $h_{j}$ and each $n$-cell of $\left(2 D^{n-1} \times[-1,1]\right)_{(M)}$ under $h_{k}$ has diameter less than $\epsilon_{i}$. For each $j$ (or $k$ ) let $T_{j}$ be a triangulation of $B_{j}$ (or $T_{k}$ be a triangulation of $\left.B_{k}\right)$ and $\sigma_{j}\left(D_{(M)}^{n}\right)\left(\right.$ or $\left.\sigma_{k}\left(2 D^{n-1} \times[-1,1]\right)_{(M)}\right)$ be a triangulation of $D^{n}$ (or $2 D^{n-1} \times[-1,1]$ ) such that $h_{j}$ (or $h_{k}$ ) is a simplicial homeomorphism. Let $\tilde{T}$ be the subdivision of $T$ containing the $T_{j}$ 's and $T_{k}$ 's as subcomplexes. Let $\tilde{a}_{\alpha(M)}$ and $\tilde{c}_{\beta(M)}$ be the polyhedral arcs in $D_{\alpha(M)}^{n}$ and in $F_{\beta(M)}^{n}$ as described in Observation 1. By very small adjustments of the given arcs we can obtain new polyhedral arcs having similar properties, but now missing the $(n-2)$-skeletons of $\sigma_{j} D_{(M)}^{n}$ or $\sigma_{k}\left(2 D^{n-1} \times[-1,1]\right)_{(M)}$. Let $a_{j \alpha}$ and $c_{k \beta}$ be the polyhedral arcs in $B_{j \alpha}$ and $F_{j \alpha}$ obtained as images of our adjusted $\tilde{a}_{\alpha(M)}$ 's and $\tilde{c}_{\beta(M)}$ 's under $h_{j}^{-1}$ and $h_{k}^{-1}$. For each $B_{j \alpha}$, let $\left\{B_{j \alpha 1}, \cdots, B_{j \alpha m(j \alpha)}\right\}$ be the collection of polyhedral 
$n$-cells obtained as images of the $n$-cells of $D_{\alpha(M)}^{n}$ under $h_{j}^{-1}$. Similarly, the $\left\{B_{k \beta 1}, \cdots, B_{k \beta m(k \beta)}\right\}$ are obtained as images of the $n$-cells of $F_{\alpha(M)}^{n}$ under $h_{k}^{-1}$. Conclusion (5) now follows.

Now $a_{j \alpha}$ (or $c_{k \beta}$ ) intersects each $B_{j \alpha i}$ (or $B_{k \beta i}$ ) in a spanning arc, except for the last cell. Also, each $a_{j \alpha}$ or $c_{k \beta}$ misses the $(n-2)$ skeleton of $\tilde{T}$. Let $P_{j \alpha}$ or $Q_{k \beta}$ be small regular neighborhoods of $a_{j \alpha}$ or $c_{k \beta}$ in $B_{j \alpha}$ or $B_{k \beta}$, respectively, and in int $B_{j}$ so that each of the $P_{j \alpha}$ and the $Q_{k \beta}$ miss the $(n-2)$-skeleton of $\tilde{T}$ and so that $G_{j \alpha}=P_{j \alpha} \cap A_{j \alpha}$ and $G_{k \beta}=Q_{k \beta} \cap C_{k \beta}$ are all $(n-1)$-cells of diameter $<\epsilon_{2}$. Let $\hat{G}_{j \alpha}=\mathrm{Bd}$ $P_{j \alpha}-\operatorname{int} G_{j \alpha}$ and let $\hat{G}_{k \beta}=\mathrm{Bd} Q_{k \beta}-\operatorname{int} G_{k \beta}$. If our regular neighborhoods are small enough and taken in a nice enough fashion, then each of $\left(B_{j \alpha i}, \hat{G}_{j \alpha} \cap B_{j \alpha i}\right)$ and $\left(B_{k \beta i}, G_{k \beta} \cap B_{k \beta i}\right)$ will be a polyhedral pair of type one or two above [2]. In a small neighborhood of each $P_{j \alpha}$ or $Q_{k \beta}$, say $\tilde{P}_{j \alpha}$, or $\tilde{Q}_{k \beta}$, we can define a PL homeomorphism $h_{j \alpha}$ or $h_{k \beta}$ carrying $\tilde{P}_{j \alpha}$ onto itself or $\tilde{Q}_{k \beta}$ onto itself so that $h_{j \alpha}=i d$ on $\operatorname{Bd} \tilde{P}_{j \alpha} \cup\left[\left(S-\operatorname{int} G_{i \alpha}\right) \cap \tilde{P}_{j \alpha}\right]$ and $h_{j \alpha}\left(G_{j \alpha}\right)=\hat{G}_{j \alpha}$ or $h_{k \beta}=i d$ on $\operatorname{Bd} \tilde{Q}_{j \alpha} \cup\left[\left(S-\right.\right.$ int $\left.\left.G_{k \beta}\right) \cap \tilde{Q}_{k \beta}\right]$ and $h_{k \beta}\left(G_{k \beta}\right)=\hat{G}_{k \beta}$. The PL homeomorphism $h: E^{n} \rightarrow E^{n}$ is obtained by defining $h$ to be the identity on $E^{n}-\left(\cup \tilde{P}_{j \alpha}\right) \cup\left(\cup \tilde{Q}_{k \beta}\right)$, equal to $h_{j \alpha}$ on $\tilde{P}_{j \alpha}$, and equal to $h_{k \beta}$ on $\tilde{Q}_{k \beta}$. If the $\tilde{P}_{j \alpha}$ 's and $\tilde{Q}_{k \beta}$ 's are small enough so as to lie in the interiors of the appropriate $B_{j}$ 's and $B_{k}$ 's, then Conclusion (6) easily follows.

6. Proof of Theorem 3. The proof of Theorem 3 will now follow quite easily from Theorems 2 and 4 as follows. Let $S, X, \epsilon^{\prime},\left\{B_{j}\right\}$, and $\left\{G_{j}\right\}$ be given as in the hypothesis of Theorem 3. We now apply Theorem 4, where $T$ is some triangulation of $E^{n}$ as required there and $\epsilon_{1}=\delta_{1}$ and $\epsilon_{2}=\delta_{2}$. Let $\tilde{T}$ be the subdivision of $T$ and $h: E^{n} \rightarrow E^{n}$ be the PL homeomorphism promised by Theorem 4 . Our desired PL homeomorphism will be a slight modification of this given $h$. We will denote the new $n$-cells in $B_{j}$ by $\tilde{B}_{j 1}, \cdots, \tilde{B}_{j m_{j}}$ and the new $(n-1)$-cells in int $G_{i}$ by $G_{i 1}, \cdots, G_{i r}$. (Recall, each $B_{j}$ was divided into two or three parts and then further divided into the appropriate $n$-cells $\left\{\tilde{B}_{j i}\right\}$ and $r_{i}$ is actually the sum of 2's and 3's.) The given $G_{i j}$ 's will be the desired $(n-1)$-cells. We now claim that if we modify the $\tilde{B}_{j i}$ 's slightly and then "shrink" them appropriately, as suggested by Observation 2, then our result will immediately follow. We will use Theorem 2 to tell us how little to shrink and modify the various $\tilde{B}_{j i}$ 's, and how to modify $h$.

That is, since $S \subset$ int $X, h=i d$ on $S-\cup G_{i j}$, and $h\left(\cup G_{i j}\right) \subset \cup$ int $B_{i}$, we have $h\left(S-\cup G_{i j}\right) \subset$ int $X$ and we can pick $0<\epsilon \leqq \epsilon^{\prime}$ so that $h\left(\cup G_{i j}\right) \subset \cup$ int $B_{j}^{\epsilon}$. For each $B_{j}$ with triangulation $\tilde{T}$ and our given $\epsilon$, we apply Theorem 2 , where $\eta<\operatorname{dist}\left(S, E^{n}-X\right)$ and $Z_{1}=Z_{2}=\cup h\left(G_{r s}\right)$, the two or three new $(n-1)$-cells in int $B_{j}$ intersecting the various $\tilde{B}_{j i}$ 's so nicely. Theorem 2 gives us an PL $\eta$ homeomorphism $h_{j}: B_{j} \rightarrow B_{j}$ such that $h_{j}=i d$ on $\operatorname{Bd} B_{j} \cup\left(\cup h\left(G_{r s}\right)\right)$ 
and the collection $h_{j}\left(\tilde{B}_{j i}\right)$ satisfy the conclusion of that theorem (the $\delta$ there is $\epsilon_{1}=\delta_{1}$ above). Since $h_{j}$ is fixed on $\cup h\left(G_{r s}\right)$, each $\left(h_{j}\left(B_{j i}\right)\right.$, $h_{j}\left(B_{j i}\right) \cap h h_{j}\left(G_{r s}\right)$ ) is still a c.c. or p.c.s. pair (so we can still shrink-in and preserve this property).

We now define $f: E^{n} \rightarrow E^{n}$ by $f=h$ on $E^{n}-\cup B_{j}$ and $f=h_{j} \circ h$ on each $B_{j}$. We observe, that since $f=h=i d$ on $E^{n}-\cup B_{j}, f=h_{j}$ on $G_{i} \cap B_{j}-\cup G_{r s}$ (where $G_{i}$ is the unique $(n-1)$-cell intersecting $B_{j}$ and $\cup G_{r s}$ is the two or three given sub $(n-1)$-cells; recall $h=i d$ on this set), and $h_{j}$ moves points less than $\eta$, it follows that $f\left(S-\cup_{i, j} G_{i j}\right) \subset$ int $X$. Also, since $h_{j}=i d$ on $\cup h\left(G_{r s}\right)$, it follows by a choice of above that $f\left(\cup_{i j} G_{i j}\right) \subset \cup$ int $B_{j}^{\epsilon}$. Thus conclusion (13) holds. We now want to shrink each $h_{j}\left(\tilde{B}_{i i}\right)$ in slightly (using the collar structure as a c.c. or p.c.s. pair) to obtain our desired disjoint $B_{j i}$ 's so that we have the required intersections with $f(S)$. We note that since $h\left(S-\cup G_{i j}\right) \subset B_{j}$ lies in $\cup B d \tilde{B}_{j i}$ and $B_{j i} \subset$ int $h_{j}\left(\tilde{B}_{j i}\right), f\left(S-\cup G_{i j}\right)$ will miss the union of the $B_{j i}$ 's. By Theorem 2, there exists a $\gamma_{j}>0$ such that any straight line intersecting $B_{j}^{\epsilon}$ also intersects some $h_{j}\left(\tilde{B}_{j i}\right)^{\gamma_{j}}$. We now shrink each $h_{j}\left(\tilde{B}_{j i}\right)$ in slightly to obtain a $B_{j i}$ with the correct intersection property so that int $B_{j i} \supset h_{j}\left(\tilde{B}_{j i}\right)^{\gamma_{j}}$. Having done this for each $j$, we finally pick one fixed $\gamma>0$ so that for each $j, B_{j i}^{\gamma} \supset h_{j}\left(\tilde{B}_{j i}\right)^{\gamma_{j}}$. It is not too difficult now to see that conclusions (4)-(14) easily follow.

Conclusion (15) is simply a consequence of hypothesis (3) and conclusion (14). That is, any straight line intersecting $X \cup\left(\cup B_{j}^{c}\right)$ must intersect some $B_{j}^{\epsilon} \supset B_{j}^{\epsilon^{\prime}}$ by (4) and hence some $B_{j i}^{\gamma}$ by (14). This completes the proof of Theorem 3 .

CoRollary 6. If $S^{2}$ is the 2-sphere in $E^{3}$ and $C$ is the Cantor subset of $S$ constructed as in the proof of Theorem 1 using the canonical modifications given by the proof of Theorem 3 , then $S^{2}$ is tame in $E^{2}$.

Proof. By [6], it will suffice to show that each complementary domain of $S^{2}$ is locally simply connected. That is, we must show that if $U$ is a complementary domain of $S^{2}$ and $p$ is any point of $S^{2}$, then given any neighborhood $W$ of $p$ in $E^{3}$ there exists a neighborhood $V$ of $p$ in $E^{3}$ such that every map of the boundary of a disk $D^{2}$ into $V \cap U$ extends to a map of $D^{2}$ into $W \cap U$. Since $S^{2}-C$ is locally flat, we only have to show that each complementary domain of $S^{2}$ is locally simply connected at points of $C$.

Let $p \in C$ and let $W$ be a neighborhood of $p$ in $E^{3}$. Recall $C=\bigcap_{i=1}^{\infty} \bigcup_{j=1}^{m_{i}} B_{i j}^{3}$. Choose $i_{0}$ large enough so that the $B_{i o j}^{3}$ containing $p$ lies in $W_{i \cdot}$ Recall $\left(B_{i 0 j}^{3}, B_{i o j}^{3} \cap S_{i_{0}}^{2}\right)$ is a c.c. or p.c.s. pair, which we can think of as the union of 3-cells $D_{1} \cup D_{2}$ or $F_{1} \cup F_{2} \cup F_{3}$ corresponding to $D_{+}^{3} \cup D_{-}^{3}$ or to $F_{0}^{3} \cup F_{-}^{3} \cup F_{+}^{3}$ as defined in $\$ 5$. Let $\tilde{B}=B_{i_{0}+1, k}^{3}$ be the 3-cell in int $B_{i 0 j}^{3}$ containing our given point $p$. Now $\tilde{B}$ lies in the interior 
of the image of one of the 3-cells above under $f_{i 0}$. Let us denote this open image by $R$. Now $R \subset$ int $B_{i_{0} j}$ and $R$ misses $S_{i_{0}+1}-$ \{some $\left.G_{i_{0}+1, j}\right\}$. Also, it follows easily that for any $k \leqq i_{0},\left(B_{i_{0} j}^{3}, B_{i_{0} j}^{3} \cap S_{i_{0}}^{2}\right)$ is PL homeomorphic to

$$
\left(B_{i 0 j}, B_{i 0 j} \cap f_{k} \circ \cdots \circ f_{i 0}\left(S_{i 0}\right)\right)=\left(B_{i 0 j}^{3}, B_{i o j}^{3} \cap S_{k+1}^{2}\right) .
$$

Moreover, if we use the nice canonical modifications as suggested in the proof of Theorem 3 (i.e., using the constructions of $\$ 5$ ), it is not too difficult to see that each component of

$$
\text { int } B_{i o j}-\left[\left(S_{k+1}^{2} \cap B_{i 0 j}\right) \cup\left(\cup\left\{B_{k+1, j} \mid B_{k+1, j} \subset \text { int } B_{i 0 j}\right\}\right)\right]
$$

is an open 3-cell, except perhaps for one which is homeomorphic to $S^{1} \times E^{2}$. This latter case occurs only if int $B_{i_{0 j}}-S_{i_{0}}$ has a component homeomorphic to $S^{1} \times E^{2}$. We now claim that any map of $\mathrm{Bd} D^{2}$ into int $\tilde{B}-S^{2}$ extends to a map of $D$ into (int $\left.B_{i 0 j}\right)-S^{2}$. Suppose $f: \operatorname{Bd} D \rightarrow($ int $\tilde{B})-S^{2}$ is any map. Choose $k>i_{0}+1$, large enough, so that $\bigcup_{i=1}^{m_{k}} B_{k j}$ misses $f(\operatorname{Bd} D)$. Then $f(\operatorname{Bd} D)$ misses $S \cup\left(\bigcup_{j=1}^{m_{k}} B_{k j}\right)$ and hence misses $S_{k} \cup\left(\bigcup_{j=1}^{m_{k}} B_{k j}\right)$ and this latter set contains $S$ (since all further modifications of $S_{k}$ leading to $S$ occur in $\cup$ int $B_{k j}$ ). Let $X=\left(S_{k} \cap B_{i_{0 j}}\right) \cup\left(\cup\left\{B_{k j} \mid B_{k j} \subset\right.\right.$ int $\left.\left.B_{i_{0 j}}\right\}\right)$. Consider int $B-X$. As above, each component of this is an open 3-cell except one, which is homeomorphic to $S^{1} \times E^{2}$. However, it is not too difficult to see that any loop in the component homeomorphic to $S^{1} \times E^{2}$ shrinks to a point in $R-X$. Therefore, either $f$ extends in int $\tilde{B}-X$ (if $f(\operatorname{Bd} D)$ lies in some component homeomorphic to an open 3-cell) or $f$ extends in $R-X$. Since each of these lie in int $B_{i o j}-S$, the result follows.

\section{REFERENCES}

1. Karol Borsuk, An example of a simple arc in space whose projection in every plane has interior points, Fund. Math., 34 (1947), 272-277.

2. Leslie C. Glaser, Geometrical Combinatorial Topology, Vol. I (Van Nostrand Reinhold Mathematical Studies, no. 27) New York: Van Nostrand Reinhold Co., 1970.

3. R. C. Kirby, On the set of non-locally flat points of a submanifold of codimension one, Ann. of Math., 88 (1968), 281-290.

4. D. R. McMillan, Jr., Taming cantor sets in $E^{n}$, Bull. Amer. Math. Soc., 70 (1964), 706-708.

5. Mark D. Meyerson, Projections of Cantor sets, simple closed curves, and spheres in $E^{3}$, to appear.

6. R. H. Bing, A surface is tame if its complement is 1-ULC, Trans. Amer. Math. Soc., 101 (1961), 294-305.

Received September 20, 1974. Research supported in part by National Science Foundation Grant No. GP 19812 Amendment \# 2. 
D. E. Bennett, Strongly unicoherent continua ............................. 1

Walter R. Bloom, Sets of p-spectral synthesis ................................ 7

R. T. Bumby and D. E. Dobbs, Amitsur cohomology of quadratic extensions: Formulas and number-theoretic examples ................. 21

W. W. Comfort, Compactness-like properties for generalized weak topological sums

D. R. Dunninger and J. Locker, Monotone operators and nonlinear biharmonic boundary value problems ...

T. S. Erickson, W. S. Martindale, 3rd and J. M. Osborn, Prime nonassociative algebras

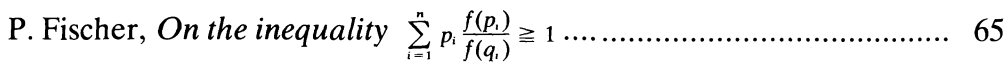

G. Fox and P. Morales, Compact subsets of a Tychonoff set ............... 75

R. Gilmer and J. F. Hoffmann, A characterization of Prüfer domains in terms of polynomials ......................................................... 81

L. C. Glaser, On tame Cantor sets in spheres having the same projection in each direction ......................................................... 87

Z. Goseki, On semigroups in which $X=X Y X=X Z X$ if and only if

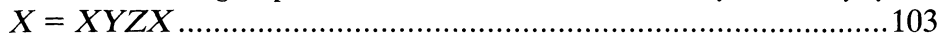

E. Grosswald, Rational valued series of exponentials and divisor

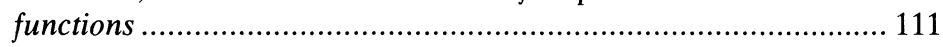

D. Handelman, Strongly semiprime rings ...................................... 115

J. N. Henry and D. C. Taylor, The $\bar{\beta}$ topology for $w^{*}$-algebras ............. 123

M. J. Hodel, Enumeration of weighted p-line arrays .......................... 141

S. K. Jain and S. Singh, Rings with quasiprojective left ideals .............. 169

S. Jeyaratnam, The diophantine equation $Y(Y+m)(Y+2 m) \times$

$$
(Y+3 m)=2 X(X+m)(X+2 m)(X+3 m) \ldots \ldots \ldots \ldots \ldots \ldots \ldots . . .183
$$

$\mathrm{R}$. Kane, On loop spaces without $p$ torsion .........................................189

Alvin J. Kay, Nonlinear integral equations and product integrals ..........203

A. S. Kechris, Countable ordinals and the analytic hierarchy, I ...........223

Ka-Sing Lau, A representation theorem for isometries of $C(X, E) \ldots \ldots . .229$

I. Madsen, On the action of the Dyer-Lashof algebra in $H_{*}(G)$..........235

R. C. Metzler, Positive linear functions, integration, and Choquet's theorem ........................................................................................ 277

A. Nobile, Some properties of the Nash blowing-up ............................297

G. E. Petersen and G. V. Welland, Plessner's theorem for Riesz conjugates 


\section{Pacific Journal of Mathematics}

\section{Vol. 60, No. $1 \quad$ September, 1975}

Donald Earl Bennett, Strongly unicoherent continua ................ 1

Walter Russell Bloom, Sets of p-spectral synthesis ................ 7

Richard Thomas Bumby and David Earl Dobbs, Amitsur cohomology of

quadratic extensions: formulas and number-theoretic examples .......

W. Wistar (William) Comfort, Compactness-like properties for generalized

weak topological sums ...................................

Dennis Robert Dunninger and John Stewart Locker, Monotone operators

and nonlinear biharmonic boundary value problems ..............

Theodore Erickson, Wallace Smith Martindale, III and J. Marshall Osborn,

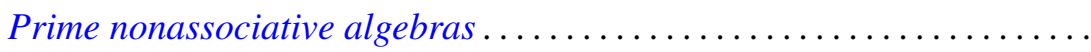

Pál Fischer, On the inequality $\sum_{i=0}^{n}\left[f\left(p_{i}\right) / f\left(q_{i}\right)\right] p_{i} \geq i \ldots \ldots \ldots \ldots \ldots$

Geoffrey Fox and Pedro Morales, Compact subsets of a Tychonoff set.......

Robert William Gilmer, Jr. and Joseph F. Hoffmann, A characterization of

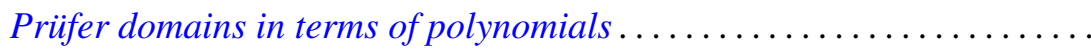

Leslie C. Glaser, On tame Cantor sets in spheres having the same projection

in each direction . ...................................

Zensiro Goseki, On semigroups in which $x=x y x=x z x$ if and only if

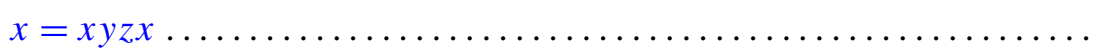

Emil Grosswald, Rational valued series of exponentials and divisor

functions.

David E. Handelman, Strongly semiprime rings

Jackson Neal Henry and Donald Curtis Taylor, The $\bar{\beta}$ topology for

$W^{*}$-algebras

Margaret Jones Hodel, Enumeration of weighted p-line arrays ...

Surender Kumar Jain and Surjeet Singh, Rings with quasi-projective left

ideals.

S. Jeyaratnam, The Diophantine equation

$$
Y(Y+m)(Y+2 m)(Y+3 m)=2 X(X+m)(X+2 m)(X+3 m) \ldots
$$

Richard Michael Kane, On loop spaces without $p$ torsion

Alvin John Kay, Nonlinear integral equations and product integrals ...

Alexander S. Kechris, Countable ordinals and the analytical hierarchy.

$I$.

Ka-Sing Lau, A representation theorem for isometries of $C(X, E)$

Ib Henning Madsen, On the action of the Dyer-Lashof algebra in $H_{*}(G)$

Richard C. Metzler, Positive linear functions, integration, and Choquet's

theorem.

Augusto Nobile, Some properties of the Nash blowing-up

Gerald E. Peterson and Grant Welland, Plessner's theorem for Riesz. 